\title{
Experimental filtering of two-, four-, and six-photon singlets from a single parametric down-conversion source
}

\author{
Magnus Rådmark, ${ }^{1}$ Marcin Wieśniak, ${ }^{2}$ Marek Żukowski, ${ }^{2}$ and Mohamed Bourennane ${ }^{1}$ \\ ${ }^{1}$ Physics Department, Stockholm University, SE-10691 Stockholm, Sweden \\ ${ }^{2}$ Institute for Theoretical Physics and Astrophysics, Uniwersytet Gdański, PL-80-952 Gdańsk, Poland
}

(Received 13 March 2009; revised manuscript received 5 May 2009; published 5 October 2009)

\begin{abstract}
Invariant entangled states remain unchanged under simultaneous identical unitary transformations of all their subsystems. We experimentally generate and characterize such invariant two-, four-, and six-photon polarization entangled states. This is done only with a suitable filtering procedure of multiple emissions of entangled photon pairs from a single source without any interferometric overlaps. We get the desired states utilizing bosonic emission enhancement due to indistinguishability. The setup is very stable and gives high interference contrasts. Thus, the process is a very likely candidate for various photonic demonstrations of quantum information protocols.
\end{abstract}

DOI: 10.1103/PhysRevA.80.040302

PACS number(s): 03.67.Mn, 03.67.Bg, 03.67.Pp

Entanglement is an essential tool in many quantum information tasks. Entangled states of two qubits proved to be useful in various quantum communication protocols such as quantum teleportation, quantum dense coding, and quantum cryptography. They are the essence of the first versions of Bell's theorem [1]. However, the expansion of quantum information science has now reached a state in which many schemes are involving multiparty processes and could require multiqubit entanglement.

There is an interesting series of multiqubit states, $\left|\Psi_{k}^{-}\right\rangle$, where $k=2,4,6$ or more. They are invariant under actions consisting of identical unitary transformations of each qubit: $U^{\otimes k}\left|\Psi_{k}^{-}\right\rangle=\left|\Psi_{k}^{-}\right\rangle$, where $U^{\otimes k}=U \otimes \cdots \otimes U$ denotes a tensor product of $k$ identical unitary operators $U[2,3]$. This property protects the states against collective noise. The states are useful, e.g., for communication of quantum information between observers who do not share a common reference frame [4]: any realignment of the receiver's reference frame corresponds to an application of the same transformation to each of the sent qubits. The states $\left|\Psi_{k}^{-}\right\rangle$can also be used for secure quantum multiparty cryptographic protocols, such as the multiparty secret sharing protocol $[5,6]$.

We generate correlations which characterize the sixphoton $\left|\Psi_{6}^{-}\right\rangle$entangled state. This is done in a six-photon interference experiment. Another six-photon interference was reported recently in [7]. To obtain graph states the authors of Ref. [7] used three pulse pumped parametric downconversion (PDC) crystals and interferometric overlaps to entangle independently emitted pairs (each from a different crystal) with each other. Schemes of this kind are generalizations of those of Ref. [8]. However, the overlaps make these schemes fragile.

In our experiment, by pulse pumping just one crystal and extracting the right order process via suitable filtering and beam splitting (the method of [9]), we observe simultaneously effects attributable to the multiphoton invariant entangled states $\left|\Psi_{2}^{-}\right\rangle,\left|\Psi_{4}^{-}\right\rangle$, and $\left|\Psi_{6}^{-}\right\rangle$. The setup has no overlaps and therefore no interferometric alignment is needed. It is strongly robust, and the output is of high fidelity with respect to the theoretical states $\left|\Psi_{k}^{-}\right\rangle$.

A simple quantum optical description of two phase matched modes of the multiphoton state that results out of a single pulse acting on a type-II PDC crystal can be put as

$$
C \sum_{n=0} \frac{1}{n !}\left[-i \alpha\left(a_{0 H}^{\dagger} b_{0 V}^{\dagger}-a_{0 V}^{\dagger} b_{0 H}^{\dagger}\right)\right]^{n}|0\rangle .
$$

The symbol $a_{0 H}^{\dagger}\left(b_{0 V}^{\dagger}\right)$ denotes a creation operator for one horizontal, $H$, (vertical, $V$ ) photon in mode $a_{0}\left(b_{0}\right)$, etc. $C$ is a normalization constant, the coupling parameter $\alpha$ is a function of pump power, nonlinearity, and length of the crystal. This is a good approximation of the actual state provided one collects the photons under conditions that allow full indistinguishability between separate two-photon emissions [9]. The first-, second-, and third-order terms in the expansion of Eq. (1) correspond to the emission of two, four, and six photons, respectively, into two spatial modes. These terms can be reinterpreted as the following superpositions of photon number states:

$$
\begin{gathered}
\left|1 H_{a_{0}}, 1 V_{b_{0}}\right\rangle-\left|1 V_{a_{0}}, 1 H_{b_{0}}\right\rangle, \\
\left|2 H_{a_{0}}, 2 V_{b_{0}}\right\rangle-\left|1 H_{a_{0}}, 1 V_{a_{0}}, 1 V_{b_{0}}, 1 H_{b_{0}}\right\rangle+\left|2 V_{a_{0}}, 2 H_{b_{0}}\right\rangle, \\
\left|3 H_{a_{0}}, 3 V_{b_{0}}\right\rangle-\left|2 H_{a_{0}}, 1 V_{a_{0}}, 2 V_{b_{0}}, 1 H_{b_{0}}\right\rangle \\
+\left|1 H_{a_{0}}, 2 V_{a_{0}}, 1 V_{b_{0}}, 2 H_{b_{0}}\right\rangle-\left|3 V_{a_{0}}, 3 H_{b_{0}}\right\rangle,
\end{gathered}
$$

where, e.g., $2 H_{a_{0}}$ and $3 H_{a_{0}}$ denote two and three horizontally polarized photons in mode $a_{0}$, respectively, etc. The secondand third-order PDC is intrinsically different than simple products of two and three entangled pairs. Due to the bosonic nature of photons, emissions of completely indistinguishable photons are more likely than the ones giving birth to photons with orthogonal polarization.

We report a joint observation, in one setup, of the correlations of the invariant two-, four- and six-photon polarization entangled states given by the following superpositions:

$$
\left|\Psi_{2}^{-}\right\rangle=\frac{1}{\sqrt{2}}(|H V\rangle-|V H\rangle),
$$




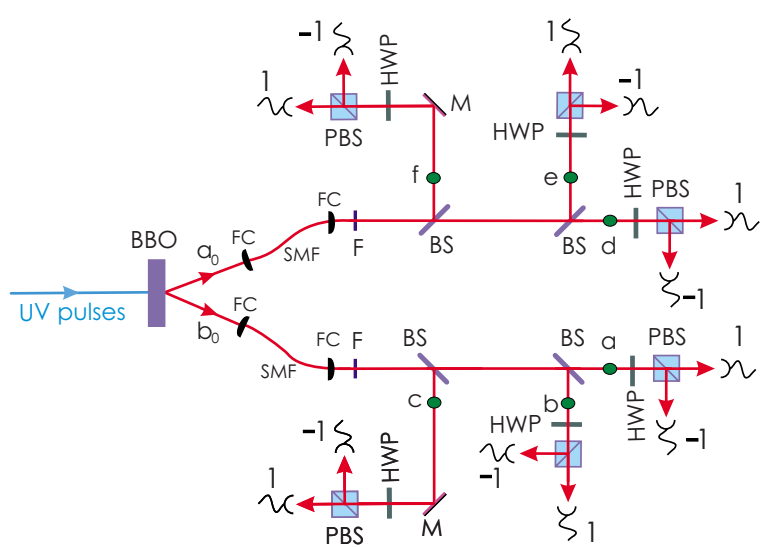

FIG. 1. (Color online) Experimental setup for generating and analyzing the six-photon polarization-entangled state. The six photons are created in third-order PDC processes in a 2-mm-thick BBO pumped by UV pulses. The intersections of the two cones obtained in noncollinear type-II PDC are coupled to SMFs wound in polarization controllers. Narrow-band interference filters (F) $(\Delta \lambda=3 \mathrm{~nm})$ serve to remove spectral distinguishability. The coupled spatial modes are divided into three modes each by $50-50 \%$ BSs. Each mode can be polarization analyzed using HWPs and a PBS. Simultaneous detection of six photons (two single-photon detectors for each mode) are being recorded by a 12 channel coincidence counter.

$$
\left|\Psi_{4}^{-}\right\rangle=\frac{2}{\sqrt{3}}\left|\mathrm{GHZ}_{4}^{+}\right\rangle-\frac{1}{\sqrt{3}}|\mathrm{EPR}\rangle|\mathrm{EPR}\rangle,
$$

and

$$
\left|\Psi_{6}^{-}\right\rangle=\frac{1}{\sqrt{2}}\left|\mathrm{GHZ}_{6}^{-}\right\rangle+\frac{1}{2}\left(\left|\bar{W}_{3}\right\rangle\left|W_{3}\right\rangle-\left|W_{3}\right\rangle\left|\bar{W}_{3}\right\rangle\right)
$$

The states in the superpositions are given by GHZ (Greenberger, Horne, Zeilinger) states

$$
\begin{gathered}
\left|\mathrm{GHZ}_{4}^{+}\right\rangle=\frac{1}{\sqrt{2}}(|H H V V\rangle+|V V H H\rangle), \\
\left|\mathrm{GHZ}_{6}^{-}\right\rangle=\frac{1}{\sqrt{2}}(|H H H V V V\rangle-|V V V H H H\rangle),
\end{gathered}
$$

the EPR (Einstein, Podolsky, Rosen) state

$$
|\mathrm{EPR}\rangle=\frac{1}{\sqrt{2}}(|H V\rangle+|V H\rangle)
$$

and finally the $W$ state

$$
\left|W_{3}\right\rangle=\frac{1}{\sqrt{3}}(|H H V\rangle+|H V H\rangle+|V H H\rangle) .
$$

The ket $|\bar{W}\rangle$ is the spin-flipped $|W\rangle$. The states in Eqs. (5)-(7) are obtained out of different orders of the PDC emission (Fig. 1), by selecting specific double, quadruple, and sixfold coincidences.

In our setup we use a frequency-doubled Ti:sapphire laser (80 $\mathrm{MHz}$ repetition rate, $140 \mathrm{fs}$ pulse length) yielding UV pulses with a central wavelength at $390 \mathrm{~nm}$ and an average power of $1300 \mathrm{~mW}$. The pump beam is focused to a $160 \mu \mathrm{m}$ waist in a 2 -mm-thick BBO ( $\beta$-barium borate) crystal. Half wave plates and two 1-mm-thick BBO crystals are used for compensation of longitudinal and transversal walk-offs. The third-order emission of noncollinear type-II PDC is then coupled to single-mode fibers (SMFs), defining the two spatial modes at the crossings of the two frequency degenerated PDC emission cones. Leaving the fibers the downconversion light passes narrow-band $(\Delta \lambda=3 \mathrm{~nm})$ interference filters $(F)$ and is split into six spatial modes $(a, b, c, d, e, f)$ by ordinary $50-50 \%$ beam splitters (BSs), followed by birefringent optics (to compensate phase shifts in the BSs). Due to the short pulses, narrow-band filters, and single-mode fibers the down-converted photons are temporally, spectrally, and spatially indistinguishable [9], see Fig. 1. The polarization is being kept by passive fiber polarization controllers. Polarization analysis is implemented by a halfwave plate (HWP), a quarter-wave plate (QWP), and a polarizing beam splitter (PBS) in each mode. The outputs of the PBSs are lead to single-photon silicon avalanche photodiodes (APDs) through multimode fibers. The APDs' electronic responses, following photo detections, are being counted by a multichannel coincidence counter with a $3.3 \mathrm{~ns}$ time window. The coincidence counter registers any coincidence event between the APDs as well as single detection events.

The states $\left|\Psi_{k}^{-}\right\rangle(k=2,4,6)$ exhibit perfect two-, four-, and six-qubit correlations. The correlation function is defined as an expectation value of the product of local polarization "Pauli" observables. If one limits the measurement to the local observables $\cos \theta_{l} \sigma_{z}^{(l)}+\sin \theta_{l} \sigma_{x}^{(l)}$ [with eigenvectors $\sqrt{1 / 2}\left(|L\rangle_{l} \pm e^{i \theta_{l}}|R\rangle_{l}\right)$ and eigenvalues \pm 1$]$, the measurements correspond to linear polarization analysis in each spatial mode, $l=a, b, c, d, e, f$. In such a case the quantum prediction for the two-photon correlation function (in modes $b$ and $d$ ) reads as $E\left(\theta_{b}, \theta_{d}\right)=-\cos \left(\theta_{b}-\theta_{d}\right)$. For the four-photon counts (in modes $a, b, d$, and $e$ ) the correlation function is given by

$$
\begin{aligned}
E\left(\theta_{a}, \theta_{b}, \theta_{d}, \theta_{e}\right)= & \frac{2}{3} \cos \left(\theta_{a}+\theta_{b}-\theta_{d}-\theta_{e}\right) \\
& +\frac{1}{3} \cos \left(\theta_{a}-\theta_{b}\right) \cos \left(\theta_{c}-\theta_{d}\right) .
\end{aligned}
$$

Finally for the six-photon events one has

$$
\begin{aligned}
E\left(\theta_{a}, \theta_{b}, \theta_{c}, \theta_{d}, \theta_{e}, \theta_{f}\right) & \\
= & -\frac{1}{2} \cos \left(\theta_{a}+\theta_{b}+\theta_{c}-\theta_{d}-\theta_{e}-\theta_{f}\right) \\
& -\frac{1}{18} \sum \cos \left(\theta_{a} \pm \theta_{b} \pm \theta_{c} \pm \theta_{d} \pm \theta_{e} \pm \theta_{f}\right),
\end{aligned}
$$

where $\Sigma$ is a sum over all possible sign sequences which contain only two positive signs, with the sign sequence in the first term proportional to $\frac{1}{2}$ excluded. Due to the invariance, the correlation functions for all measurements around any single great circle of the Bloch sphere look the same.

Figure 2 shows three experimentally observed two-photon correlation functions, $E\left(\theta_{b}, \theta_{l}\right)$, where $l=d, e, f$. The setting 

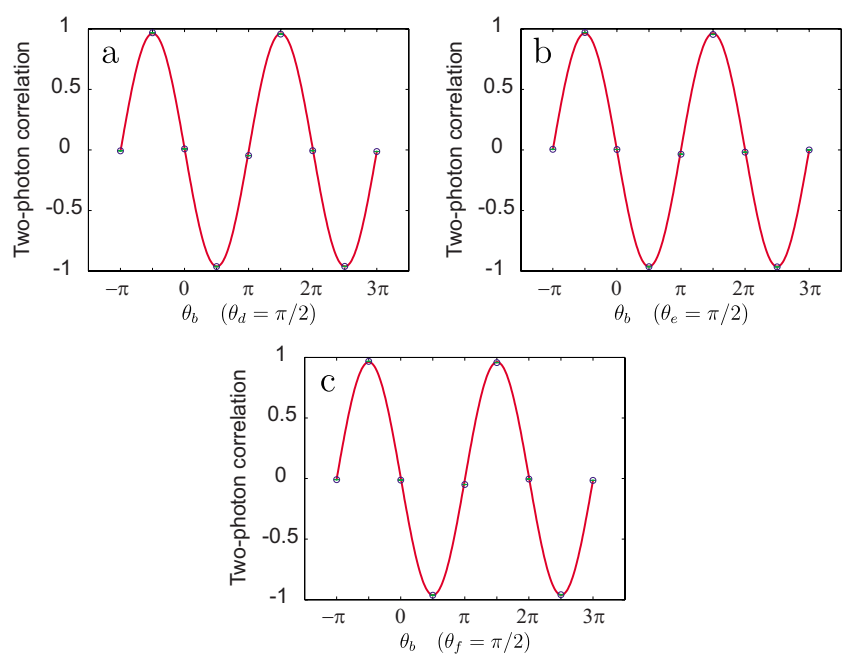

FIG. 2. (Color online) Two-photon polarization correlation function. Modes $a, d, e$, and $f$ are analyzed in $(D, A)$ polarization basis and mode $b$ analysis basis is varied around the equator of the Bloch sphere $\left[\sigma_{z} \cos \left(\theta_{b}\right)+\sigma_{x} \sin \left(\theta_{b}\right)\right]$. The three figures correspond to different implementations of the state using different modes $(b d, b e$, and $b f$ ). The solid lines show sinusoidal fits to the experimental data with an average visibility of $V_{2}=96.2 \% \pm 0.3 \%$. The average measurement time for each data point was $77 \mathrm{~h}$; the statistical errors are negligible on the presented scale.

$\theta_{b}$ is varied, while the other analyzer is fixed at $\theta_{l}=\pi / 2$. This corresponds to diagonal, antidiagonal $(D, A)$ linear polarization basis [10]. A sinusoidal least-squares fit was made to the data. The average visibility, defined here as the average amplitude of the three fits, is $V_{2}=96.2 \% \pm 0.3 \%$. Figure 3 shows how six experimentally observed four-photon correlation functions $E\left(\theta_{b}, \theta_{l}, \theta_{m}, \theta_{n}\right)$ depend on $\theta_{b}$. The other analyzers were fixed at $\theta_{l}=\theta_{m}=\theta_{n}=\pi / 2$, where $l=a, c, m=d, e$, and $n=e, f$. The average value of the six visibilities is $V_{4}$ $=91.9 \% \pm 0.5 \%$. Finally, Fig. 4 shows similar data for the experimentally observed six-photon correlation function $E\left(\theta_{a}, \theta_{b}, \theta_{c}, \theta_{d}, \theta_{e}, \theta_{f}\right)$. Again $\theta_{b}$ was varied with the other five analyzers fixed at $\theta_{a}=\theta_{c}=\theta_{d}=\theta_{e}=\theta_{f}=\pi / 2$. The value of the visibility is $V=84 \% \pm 3 \%$. In Table I we present all experimentally obtained two-, four-, and six-photon visibilities. Also the average count rates for each set of modes are given. With the current rates the noise contribution due to higherorder emissions is small, as, e.g., the ratio of six-photon events to four-photon events is small.

We have compared the observed visibilities with theoretical predictions [11]. To estimate maximal predictable visibilities one can use a less simplified description of the twophoton state emitted by PDC events and replace in Eq. (1) $\left(a_{0 H}^{\dagger} b_{0 V}^{\dagger}-a_{0 V}^{\dagger} b_{0 H}^{\dagger}\right)$ by

$$
\begin{aligned}
\int d t & \int d \omega_{0} \int d \omega_{1} \int d \omega_{2} f\left(\omega_{1}\right) f\left(\omega_{2}\right) g\left(\omega_{0}\right) e^{i \omega t} \\
& \times \Delta\left(\omega_{0}-\omega_{1}-\omega_{2}\right)\left[a_{0 H}^{\dagger}\left(\omega_{1}\right) b_{0 V}^{\dagger}\left(\omega_{2}\right)-a_{0 V}^{\dagger}\left(\omega_{1}\right) b_{0 H}^{\dagger}\left(\omega_{2}\right)\right] .
\end{aligned}
$$

This approach is rich enough to take into account the frequency phase matching conditions. The creation operators
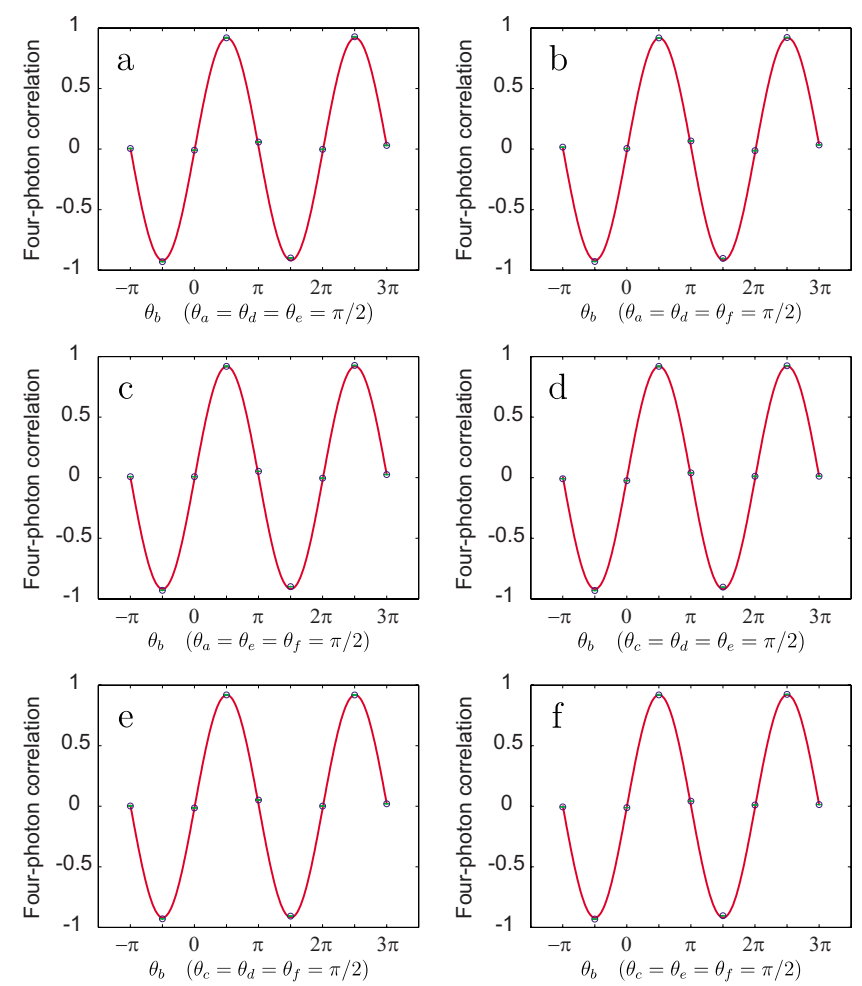

FIG. 3. (Color online) Four-photon polarization correlation functions of $\left|\Psi_{4}^{-}\right\rangle$. Modes $a, c, d, e$, and $f$ are analyzed in $(D, A)$ polarization basis and mode $b$ analysis basis is rotated around the equator of the Bloch sphere $\left(\hat{\sigma}_{z} \cos \theta_{b}+\hat{\sigma}_{x} \sin \theta_{b}\right)$. The figures correspond to different implementations of the state using different modes (abde, abdf, abef, bcde, bcdf, and bcef). The solid lines show sinusoidal fits to the experimental data with an average visibility of $V_{4}=91.9 \% \pm 0.5 \%$. The average measurement time for each data point was $77 \mathrm{~h}$; the statistical errors are negligible on the presented scale.

depend additionally on frequencies and obey $\left[a_{0 X}(\omega), a_{0 X^{\prime}}\left(\omega^{\prime}\right)\right]=\delta_{X X^{\prime}} \delta_{X X^{\prime}}\left(\omega-\omega^{\prime}\right)$, etc. The function $f(\omega)$ represents the shape of the filter transmission profiles, and $g\left(\omega_{0}\right)$ represents the frequency profile of the pump pulse. If one chooses $f(\omega)=\exp \left\{-\left[\left(\omega_{f}-\omega\right) /\left(2 \sigma_{f}\right)\right]^{2}\right\}$ and $g(\omega)=\exp \left\{-\left[\left(\omega_{p}-\omega\right) /\left(2 \sigma_{p}\right)\right]^{2}\right\}$, where $\sigma_{p}$ and $\sigma_{f}$ are the full width at half maximum (FWHM) bandwidths of the pump and the filters, and $\omega_{f}=\omega_{p} / 2$, the following formulas for the maximal theoretical visibility as a function of the ratio $r=\sigma_{f} / \sigma_{p}$ can be reached [11]. For the four-photon process, $V_{4}^{\text {temp }}=\sqrt{1+2 r^{2}} /\left(1+r^{2}\right)$, and for the six-photon process, $V_{6}^{t e m p}=\left(1+2 r^{2}\right) /\left[\left(1+r^{2} / 2\right)\left(1+3 r^{2} / 2\right)\right]$. In our experiment we used $r_{\text {exp }}=\Delta \lambda_{f} /\left(4 \Delta \lambda_{p}\right)=0.76 \pm 0.07$, corresponding to $V_{4}=93 \% \pm 2 \%$ and $V_{6}=90 \% \pm 3 \%$. The actual measured values of the visibility for two-, four-, and six-photon interference are very close to the predicted ones, see Table I. Thus, the fact that our setup use only filtering and beam splitting has interferometric advantages. In other words the obtained four- and six-particle visibilities are almost as high as one would predict for the ratio $r_{\text {exp }}$.

The high visibility has the following consequences. With just a part of our data one can use the simplest of the "experimentally friendly" entanglement indicators introduced in [12]. It guarantees that an $N$-qubit state is entangled if the 


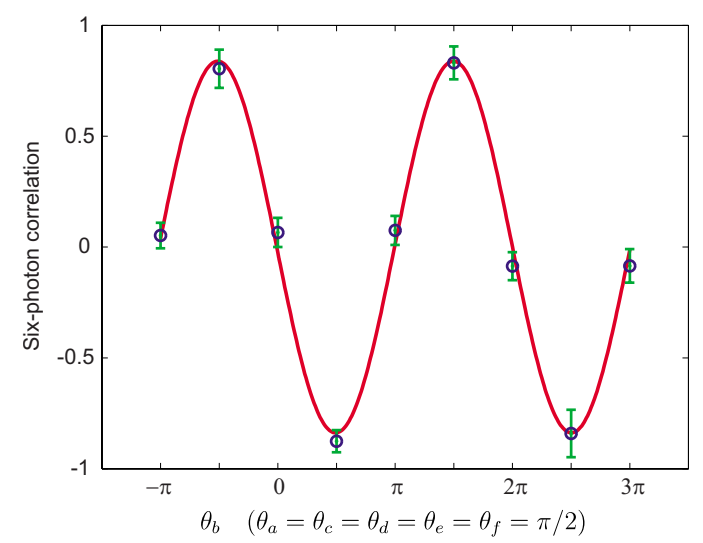

FIG. 4. (Color online) Six-photon polarization correlation function. Modes $a, c, d, e$, and $f$ are analyzed in $(D, A)$ polarization basis and mode $b$ analysis basis is varied around the equator of the Bloch sphere $\left[\sigma_{z} \cos \left(\theta_{b}\right)+\sigma_{x} \sin \left(\theta_{b}\right)\right]$. The solid line shows a sinusoidal fit to the experimental data with a visibility of $84 \% \pm 3 \%$. The average measurement time for each data point was $77 \mathrm{~h}$.

norm of the $N$-particle correlation tensor is higher than 1 . For the $\left|\Psi_{2}^{-}\right\rangle$we take just $T_{x x}, T_{y y}, T_{z z}$; for $\left|\Psi_{4}^{-}\right\rangle$we again take just $T_{x x x x}, T_{y y y y}, T_{z z z z} ;$ and for $\left|\Psi_{6}^{-}\right\rangle$we take the components $T_{x x x x x x}, T_{\text {yyyyyy}}$, and $T_{z z z z z z}$. With our data we have obtained the values $2.785 \pm 0.007,2.517 \pm 0.011$, and $2.29 \pm 0.14$, respectively, for each of these cases. The entanglement threshold is violated by of 242, 133, and 9.3 standard deviations. Additionally, according to the criteria given in [13] the state cannot be described by a local realistic model if the sum of squares of two of the listed components is above 1 . This is again achieved by our data: for two particles we obtain $T_{x x}^{2}$ $+T_{y y}^{2}=1.837 \pm 0.006$ (exceeding 1 by 151 standard deviations), for four particles we get $T_{x x x x}^{2}+T_{y y y y}^{2}=1.646 \pm 0.009$ (exceeding 1 by 75 standard deviations), and for six particles we have $T_{x x x x x x}^{2}+T_{\text {yyyyy }}^{2}=1.52 \pm 0.11$ (exceeding 1 by 4.5 standard deviations). Thus can the states directly (that is without still enhancing its fidelity) be utilized in classical threshold beating communication complexity protocols [14].

In summary, we have experimentally demonstrated a versatile source of multiphoton entanglement allowing two-, four-, and six-photon high visibility interference due to entanglement. Our experiment is based upon a single pulsed PDC source and a simple filtering procedure, and thus we avoid interferometric alignment problems and the setup is very stable. We utilize the bosonic emission enhancement occurring in the emission of multiple photon pairs in PDC, thus the process is not entirely spontaneous. This stimulated
TABLE I. Visibilities and count rates of the invariant states $\left|\Psi_{k}^{-}\right\rangle$ with $k=2,4,6$.

\begin{tabular}{cccc}
\hline \hline & & $\begin{array}{c}\text { Visibility } \\
(\%)\end{array}$ & Count rate \\
\hline 2 & Modes & $96.2 \pm 0.4$ & $4604 \mathrm{~s}^{-1}$ \\
2 & $b, d$ & $96.3 \pm 0.6$ & $3814 \mathrm{~s}^{-1}$ \\
2 & $b, f$ & $96.2 \pm 0.4$ & $7916 \mathrm{~s}^{-1}$ \\
4 & $a, b, d, e$ & $91.9 \pm 1.4$ & $0.6 \mathrm{~s}^{-1}$ \\
4 & $a, b, d, f$ & $91.8 \pm 1.1$ & $1.3 \mathrm{~s}^{-1}$ \\
4 & $a, b, e, f$ & $91.9 \pm 1.4$ & $1.1 \mathrm{~s}^{-1}$ \\
4 & $b, c, d, e$ & $91.9 \pm 1.4$ & $1.2 \mathrm{~s}^{-1}$ \\
4 & $b, c, d, f$ & $91.8 \pm 0.9$ & $2.6 \mathrm{~s}^{-1}$ \\
4 & $b, c, e, f$ & $92.0 \pm 1.2$ & $2.1 \mathrm{~s}^{-1}$ \\
6 & $a, b, c, d, e, f$ & $84 \pm 3$ & $2.6 \mathrm{~h}^{-1}$ \\
\hline \hline
\end{tabular}

emission is also resulting in a higher rate of multiphoton emission, given the same pump conditions, than in the case of other schemes relying on the use of several crystals and only utilizing spontaneous PDC emission in each crystal. Furthermore such multicrystal setups need interferometric overlaps to achieve multiphoton entanglement, which leads to difficulties in obtaining good and stable interference. To increase the count rates in our setup a number of factors could be improved. These include higher pump power, more efficient crystals with narrower emission bandwidth, better coupling to single-mode fiber, and more efficient singlephoton detectors.

This multiphoton source has been used to generate multipartite entangled singlet states by dividing the two spatial emission modes into six modes and hence keeping the high coherence of the source to the final states. The states that we observed are of a high fidelity and they are invariant with respect to simultaneous identical (unitary) transformations of all qubits. This makes them particularly useful for decoherence-free communication. We would like to note that the achieved interference contrast is high enough for our source to be used in two-, four-, and six-party demonstrations of quantum reduction in communication complexity in some joint computational tasks and for secret sharing, as well as in many other quantum informational protocols.

This work was supported by the Swedish Research Council (VR). M.Ż. was supported by the Wenner-Gren Foundations and by the EU program QAP (Qubit Applications, Grant No. 015858).
[1] J.-W. Pan et al., e-print arXiv:0805.2853.

[2] M. Bourennane et al., Phys. Rev. Lett. 92, 107901 (2004).

[3] A. Cabello, Phys. Rev. A 75, 020301(R) (2007).

[4] S. D. Bartlett et al., Phys. Rev. Lett. 91, 027901 (2003).

[5] M. Hillery et al., Phys. Rev. A 59, 1829 (1999).

[6] S. Gaertner et al., Phys. Rev. Lett. 98, 020503 (2007).

[7] C.-Y. Lu et al., Nat. Phys. 3, 91 (2007).

[8] A. Zeilinger et al., Phys. Rev. Lett. 78, 3031 (1997).
[9] M. Zukowski et al., Ann. N.Y. Acad. Sci. 755, 91 (1995).

[10] S. Gaertner et al., Appl. Phys. B: Lasers Opt. 77, 803 (2003).

[11] W. Laskowski et al., J. Phys. B 42, 114004 (2009).

[12] P. Badziag et al., Phys. Rev. Lett. 100, 140403 (2008).

[13] M. Zukowski and C. Brukner, Phys. Rev. Lett. 88, 210401 (2002).

[14] C. Brukner et al., Phys. Rev. Lett. 92, 127901 (2004). 